\title{
Efecto antioxidante de la melatonina durante la conservación de semen de cerdo
}

\author{
Flores, C.; Meléndez, C.; Mendoza, C.; Márquez, Y.; Vilanova, L.T.
}

Unidad de Investigación en Ciencias Funcionales Dr. Haity Moussatché (UNIHM), Facultad de Ciencias Veterinarias, Universidad Centroccidental “Lisandro Alvarado" (UCLA), Barquisimeto (Lara, Venezuela).

E-mail: caf06@hotmail.com

\begin{abstract}
Resumen
Flores, C.; Meléndez, C.; Mendoza, C.; Márquez, Y.; Vilanova, L.T.: Efecto antioxidante de la melatonina durante la conservación de semen de cerdo. Rev. vet. 29: 1, 13-17, 2018. Durante los procesos de conservación espermática se incrementa la producción de radicales libres y se ha planteado la adición de antioxidantes para mejorar la calidad seminal durante el almacenamiento. Ello asume importancia en especies como el cerdo, donde se ha relacionado el estrés oxidativo al daño del espermatozoide durante la conservación, lo que afecta su capacidad fecundante. Esta investigación tuvo como objetivo determinar el efecto antioxidante de la melatonina durante la conservación de semen de cerdos a $16^{\circ} \mathrm{C}$. Las muestras seminales se tomaron de verracos de 7-18 meses de edad. La recolección del semen se realizó con mano enguantada. Las muestras seminales con parámetros óptimos fueron diluidas y divididas en alícuotas: control (con diluyente), control con vehículo (diluyente y etanol) y semen con melatonina $(1,25 \mathrm{mM})$. Luego de la preparación de las alícuotas (día 0) y al día 1 de conservación a $16^{\circ} \mathrm{C}$, se evaluaron indicadores de lipoperoxidación como dienos conjugados (DC, extracción con isopropanol) y malondialdehído; la actividad enzimática de la superoxido dismutasa (SOD) se determinó mediante un kit comercial. Los resultados fueron analizados mediante ANOVA, con paquete estadístico SPSS 15.0 para Windows con $\mathrm{p} \leq 0,05$. Los niveles de DC en las muestras con melatonina se incrementaron al día 0 y 1 de conservación, de manera significativa $(\mathrm{p} \leq 0,05)$ con respecto al grupo control y con el vehículo, mientras que las concentraciones de malondialdehído al día 1 de conservación en las muestras con melatonina presentaron valores significativamente menores $(\mathrm{p} \leq 0,05)$ con respecto al grupo control, con mayor actividad de la SOD $(\mathrm{p} \leq 0,05)$. Se concluye que la adición de melatonina al semen de cerdo diluido tiene efecto antioxidante durante la conservación espermática a $16^{\circ} \mathrm{C}$.
\end{abstract}

Palabras clave: cerdo, conservación del semen, antioxidante, melatonina.

\begin{abstract}
Flores, C.; Meléndez, C.; Mendoza, C.; Márquez, Y.; Vilanova, L.T.: Antioxidant effect of the melatonin during the conservation of boar semen. Rev. vet. 29: 1, 13-17, 2018. During the processes of sperm preservation the free radical production increases, and it has been suggested that the addition of antioxidants improve semen quality during storage, mainly in species such as pork, where this has been linked to oxidative stress and sperm damage during storage, which affects their fertilizing capacity. The goal of this research is to determine the antioxidant effect of the melatonin during the conservation of semen of boars to $16^{\circ} \mathrm{C}$. Semen samples were taken of boars 7-18 months old. Semen collection was made with gloved hand. Semen samples with optimal parameters were diluted and divided into aliquots: control (i.e., diluent), control with vehicle (diluent and ethanol) and semen with melatonin (1.25 mM). After the preparation of aliquots (day 0 ) and on day 1 of conservation at $16^{\circ} \mathrm{C}$, lipoperoxidation indicators such as conjugated dienes (DC, isopropanol extraction) and malondialdehyde, were evaluated, The enzymatic activity of dismutase superoxide (SOD) was determined by commercial kit. The results were analyzed by ANOVA, with package statistical SPSS 15.0 for Windows with $\mathrm{p} \leq 0.05$. Them levels of conjugate dienes (DC) in them samples with melatonin is increase to the day 0 and 1 of conservation, of way significant $(p \leq 0,05)$ with regard to the control group and with the vehicle, while the concentrations of malondialdehyde to the day 1 of conservation in them samples with melatonin presented values significantly $(p \leq 0,05)$ minor with respect to the control group, with greater activity of the SOD $(p \leq 0,05)$.
\end{abstract}


In conclusion, the addition of melatonin in dilute boar semen has an antioxidant effect during sperm storage to $16^{\circ} \mathrm{C}$.

Key words: boar, conservation of semen, antioxidant, melatonin.

\section{INTRODUCCIÓN}

En el cerdo la preservación del semen es limitada debido a que los espermatozoides de esta especie son especialmente sensibles a temperaturas por debajo de $15^{\circ} \mathrm{C}$, las cuales generan daños en la membrana plasmática y en el acrosoma, alterando la viabilidad y funcionalidad de la célula espermática ${ }^{24}$.

Tales efectos de la conservación seminal se han relacionado con la producción excesiva de radicales libres (RL) en el espermatozoide ${ }^{7}$ que a altas concentraciones generan estrés oxidativo (EO), causando alteraciones celulares como la lipoperoxidación de la membrana plasmática, situación a la que el espermatozoide es particularmente susceptible, debido a las altas concentraciones de ácidos grasos polinsaturados en la membrana plasmática ${ }^{28}$.

Si bien los espermatozoides son sensibles al estrés oxidativo, también están equipados con un sistema antioxidante en el plasma seminal e intracelular ${ }^{7}$, los cuales están armónicamente integrados ${ }^{1,6}$ para contrarrestar los efectos tóxicos de las especies reac tivas de oxígeno (EROs) ${ }^{7}$.

Dentro del sistema antioxidante enzimático del semen destaca la superóxido dismutasa (SOD) ${ }^{10}$, y se ha podido constatar que a lo largo de la curva de enfriamiento del proceso de criopreservación y en el postdescongelamiento, la concentración de esta enzima disminuye, dejando expuesto a los espermatozoides a la acción de los RL ${ }^{15}$.

En tal sentido, en los últimos años se han realizado estudios con el uso de diferentes antioxidantes (vitamina $\mathrm{C}$ y vitamina $\mathrm{E}$ ) en los diluyentes, para evitar el efecto deletéreo de los RL durante la conservación espermática en diferentes especies ${ }^{7}$.

Se ha demostrado la actividad antioxidante de la melatonina, con efectos superiores a otros antioxidantes (E, C y N-acetilcisteína) a nivel de células hepáticas y una de sus principales cualidades es que varios de los metabolitos, como el N1-acetil-N2-formil 5-methoxykynuramine y N1-acetil-5-methoxykynuramine, que resultan de la interacción de la melatonina con el radical hidroxilo, funcionan también como eliminadores de RL, es decir, que una molécula de melatonina puede neutralizar hasta 3 radicales libres ${ }^{22,23,29}$.

En base a estos antecedentes, la presente investigación planteó como objetivo determinar el efecto de la adición de melatonina como antioxidante durante la conservación de semen de cerdo diluido.

\section{MATERIAL Y MÉTODOS}

Población y muestra. El estudio se llevó a cabo en la UCLA (Barquisimeto, Venezuela). Las 20 muestras de semen para el estudio, fueron tomadas de verracos de 7-18 meses de edad y de razas Landrace, Large White y Duroc, procedentes de la granja de reproductores de Inversiones Porcinas C.A., ubicada en la carretera vieja Yaritagua-Barquisimeto, estado Yaracuy-Venezuela. Los machos reproductores seleccionados como donadores de semen eran animales sanos, mantenidos en condiciones óptimas de salud, en un hábitat limpio y con una nutrición balanceada, siguiendo lo establecido en el Código de Ética para la Vida de la República Bolivariana de Venezuela (2010) en su segunda parte capítulo 3.

Toma de muestra. La recolección de la muestra seminal se realizó mediante masaje peneano con mano enguantada en un potro adecuado para tal fin. Luego de obtener las muestras experimentales, fueron analizadas en el laboratorio de la granja y las que cumplían con los criterios de selección seminal para los programas de inseminación artificial, fueron diluidas para su conservación a $16^{\circ} \mathrm{C}$ con diluyente comercial para semen de cerdo $\left(\mathrm{MR}-\mathrm{A}^{\circledR}\right)$, tomando en cuenta el volumen y la densidad óptica del eyaculado.

Manejo de la muestra. De cada eyaculado seleccionado al azar, el primer día (día 0) se prepararon 3 alícuotas de $5 \mathrm{ml}$, las cuales fueron etiquetadas como control (solo diluyente MR-A ${ }^{\circledR}$ ), control con el vehículo (etanol), y la alícuota con melatonina (Sigma ${ }^{\circledR}$ ) a 1,25 $\mathrm{mM}$, esta última diluida y agregada a razón de $20 \mu \mathrm{l}$ por $\mathrm{ml}$ de semen diluido.

Determinación de indicadores hepáticos de lipoperoxidación. Se midieron indicadores de estrés oxidativo como los dienos conjugados (DC), que son los productos iniciales del proceso de lipoperoxidación. Fueron extraídos con isopropanol y valorados por la técnica de Wallin ${ }^{30}$. El malondialdehído (MDA), producto final del proceso de lipoperoxidación, se determinó mediante el test para sustancias reaccionantes con el ácido 2-tiobarbitúrico acorde al método de Ohkawa ${ }^{19}$. La actividad enzimática de superóxido dismutasa (SOD) se obtuvo mediante un kit comercial $\left(\right.$ Cayman $\left.{ }^{\circledR}\right)$ y los resultados fueron expresados en $U$ de $\mathrm{SOD} / \mathrm{mg}$ de proteínas. Las proteínas totales se determinaron mediante el kit Bio Rad (Richmond, CA, EEUU.) basado en el método de Bradford ${ }^{3}$. 
Técnicas de procesamiento y análisis de datos. Los datos fueron analizados mediante paquete estadístico SPSS 15.0 para Windows y se realizó ANOVA de un factor con un criterio de clasificación $(p \leq 0,05)$.

\section{RESULTADOS}

Al evaluar los niveles de DC y MDA como indicadores de estrés oxidativo, se pudo evidenciar que en el semen con melatonina a 1,25 mM, los niveles de DC el día 0 y 1 de conservación presentaron elevación significativa $(p \leq 0,05)$ de las concentraciones de este biomarcador, con respecto al grupo control y con vehículo (Figura 1).

Por su parte, los niveles de MDA en las muestras con melatonina (Figura 2) presentaron niveles menores al día 0 , cuya reducción se hizo significativa el día 1 de conservación $(\mathrm{p} \leq 0,05)$.

Al evaluar la actividad enzimática total de la SOD se pudo evidenciar que el grupo de muestras con melatonina presentó mayor actividad enzimática durante

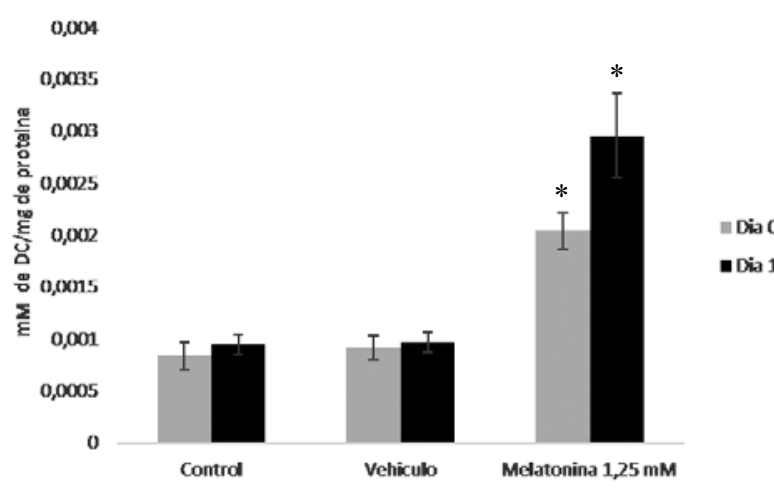

Figura 1. Niveles de DC en semen diluido de cerdos de 7-18 meses de edad, días 0 y 1 de conservación a $16^{\circ} \mathrm{C}$ con melatonina a $1,25 \mathrm{mM}$. *Evidencia diferencias significativas de las muestras con melatonina al $1,25 \mathrm{mM}$ con respecto al control y vehículo (días 0 y 1 ), $\mathrm{p} \leq 0,05$.

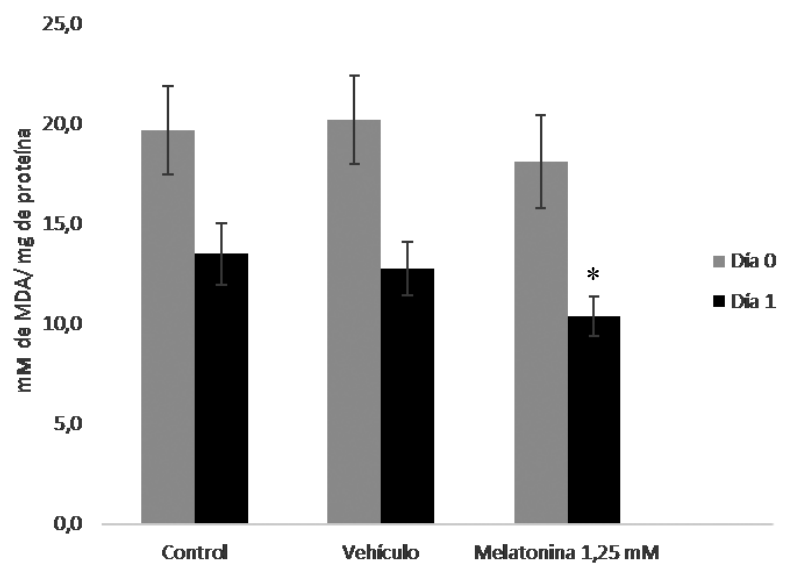

Figura 2. Niveles de MDA en semen diluido de cerdos de 7-18 meses de edad, el día 0 y 1 de conservación a $16^{\circ} \mathrm{C}$ con melatonina a $1,25 \mathrm{mM}$. *Evidencia diferencias significativas de las muestras con melatonina al $1,25 \mathrm{mM}$ con respecto a las muestras control y vehículo, al día 1 de conservación $(\mathrm{p} \leq 0,05)$. la conservación, con diferencias significativas el día 1 $(p \leq 0,05)$, con respecto al grupo control y con vehículo (Figura 3).

Estos resultados demuestran que la melatonina a $1,25 \mathrm{mM}$ en semen de cerdo diluido tiene efecto antioxidante durante la conservación a $16^{\circ} \mathrm{C}$.

\section{DISCUSIÓN}

La criopreservación espermática es una biotecnología de gran trascendencia, debido a que tiene un papel relevante en la conservación y difusión de recursos genéticos. Sin embargo, en el cerdo esta técnica no ha tenido tanto éxito como el obtenido en bovinos, por lo tanto el manejo del semen de cerdo para reproducción asistida es principalmente en fresco o conservado mediante refrigeración a $16{ }^{\circ} \mathrm{C}^{12}$.

Por otro lado, la conservación de semen, es un procedimiento que genera $\mathrm{EO}$ al incrementar los niveles de EROS y al disminuir la disponibilidad de los antioxidantes endógenos ${ }^{2}$. Este evento puede causar daño oxidativo en los espermatozoides durante el almacenamiento y ser la causa de disminución de la movilidad y fertilidad, por lo que se ha evaluado utilizar antioxidantes exógenos para mantener la calidad seminal durante su conservación ${ }^{18}$.

En esta investigación la melatonina a una concentración 1,25 Mm tuvo acción antioxidante en el semen de cerdo los días 0 y 1 de conservación a $16^{\circ} \mathrm{C}$, con aumento de la actividad enzimática de SOD y al evidenciar una interrupción del proceso de la lipoperoxidación, que pudiera ocurrir por la interacción del radical lipoperoxilo con la melatonina, de esta manera el proceso de lipoperoxidación entra en fase de terminación, lo que promueve el aumento de los niveles de DC y la disminución de MDA.

La mayoría de los estudios que evalúan EO en semen conservado, no determinan DC, lo que dificulta contrastar los resultados obtenidos. En el caso de los niveles de MDA, en esta investigación se obtuvo un resultado similar al reportado por otros investigadores en semen de ciervo tratado con melatonina ${ }^{8}$, la cual redu-

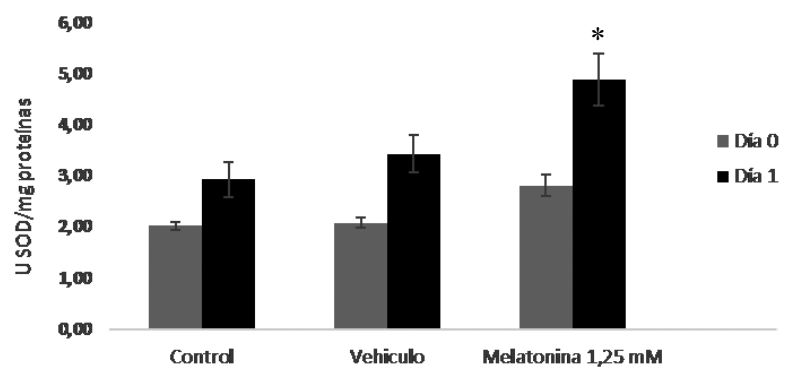

Figura 3. Niveles de SOD total en semen diluido de cerdos de 7-18 meses de edad, el día 0 y 1 de conservación a $16^{\circ} \mathrm{C}$ con melatonina a $1,25 \mathrm{mM}$. *Muestra diferencias significativas del semen con melatonina a $1,25 \mathrm{mM}$ en el día 1 de conservación con respecto a las muestras control y vehículo $(\mathrm{p} \leq 0,05)$. 
jo los niveles de MDA. De igual forma existen reportes en semen humano, en el cual la melatonina disminuyó significativamente en un $63 \%$ el MDA en comparación con el grupo control ${ }^{13}$.

Por otra parte, en semen bovino almacenado in vitro hasta $30 \mathrm{~h}$, la melatonina demostró tener efectos protectores sobre los parámetros espermáticos relacionados con disminución en la producción de MDA, incluso en diferentes épocas del año ${ }^{21}$.

Sin embargo, en espermatozoides de gallos indígenas de Irán, la melatonina a concentraciones de 3 y 6 $\mathrm{mM}$, disminuyó significativamente la viabilidad de los espermatozoides en comparación al grupo control, con niveles máximos de MDA al usar melatonina a $6 \mathrm{mM}$ ${ }^{17}$. De igual manera, en otros estudios la melatonina a 1 Mm en semen de cerdo no obtuvo efecto antioxidante, por el contrario aumentó los niveles de RL ${ }^{16}$.

La variabilidad encontrada entre las especies en respuesta a la adición de melatonina, pudiera deberse a la variación en la susceptibilidad a la lipoperoxidación que presentan los espermatozoides de cada una de ellas. Esta diferencia se ha relacionado a la proporción de ácidos grasos poliinsaturados (PUFA) en la membrana del espermatozoide ${ }^{25}$ que juega un rol importante en las modificaciones fisicoquímicas que ocurren durante la fecundación ${ }^{14}$.

En el caso de las aves, la clase de PUFA que predomina en la membrana espermática es del tipo n-6, específicamente $22 \mathrm{C}: 4$ y $20 \mathrm{C}: 4{ }^{27}$, mientras que en la mayoría de los mamíferos predominan (más del 60\% del total de ácidos grasos) los de la serie n-3 de cadena larga altamente insaturados, como el 22:5, 22:6 y 20:4 que poseen alto riesgo de ser oxidados ${ }^{4}$.

Así el 22C:6 se encuentra en altas cantidades en la membrana de los espermatozoides del cerdo y se ha podido constatar en esta especie que, durante la conservación, estos fosfolípidos van disminuyendo en proporción en la membrana de los espermatozoides asociados al proceso de lipoperoxidación ${ }^{5}$.

Estos elementos pudieran afectar la respuesta a la adición de melatonina al semen durante la conservación entre las especies, de allí la importancia de que se realicen estudios previos, antes de adicionar antioxidante al semen para la criopreservación, con el propósito de determinar la dosis correcta para mantener niveles adecuados de MDA durante la conservación en cada una de las especies en particular, lo cual es de importancia debido a que altos niveles de este biomarcador de estrés oxidativo se han asociado con disminución de la capacidad fecundante que conlleva finalmente a infertilidad masculina ${ }^{7}$.

En el caso de la actividad enzimática de la SOD otros investigadores obtuvieron resultados similares a los del presente trabajo, al determinar que la melatonina a concentraciones de $0,1 \mathrm{mM}$ elevó la actividad de la SOD en semen de búfalo luego de ser conservado en nitrógeno líquido $\left(-196^{\circ} \mathrm{C}\right)$ por $24 \mathrm{~h}$ en comparación con el control ${ }^{9}$.
Resultados similares se han obtenido en seres humanos con muestras espermáticas frescas, en caneros con semen congelado y en verracos con semen refrigerado, demostrando que la melatonina protege a los espermatozoides contra el estrés oxidativo ${ }^{11,26}$.

Igualmente, en bovinos la melatonina eleva la actividad enzimática de la SOD ${ }^{21}$. La melatonina puede incluso preservar la actividad de enzimas antioxidantes a nivel intracelular durante la conservación, aún en diferentes épocas del año ${ }^{20}$.

En base a los resultados se puede concluir que la melatonina a $1,25 \mathrm{mM}$, tiene efecto antioxidante (días 0 y 1) en semen de cerdo conservado a $16^{\circ} \mathrm{C}$.

Agradecimientos: A la Unidad de Investigación en Ciencias Funcionales Dr. Haity Moussatché y al Consejo de Desarrollo Científico, Humanístico y Tecnológico (CDCHT-UCLA) por su apoyo para la realización de esta investigación.

\section{REFERENCIAS}

1. Aitken RJ, Krausz C. 2001. Oxidative stress, DNA damage and the Y chromosome. Reprod 122: 497-506.

2. Ball BA. 2008. Oxidative stress, osmotic stress and apoptosis: Impacts on sperm function and preservation in the horse. Anim Reprod Sci 107: 257-267.

3. Bradford M. 1976. A rapid and sensitive method for the quantities of microgram of protein utilizing the principle of protein-dye binding. Anal Biochem 72: 248-254.

4. Castellini C, Cardinali R, Dal Bosco A, Minelli A, Camici O. 2006. Lipid composition of the main fractions of rabbit semen. Theriogenology 65: 703-12.

5. Cerolini S, Maldjian A, Surai P, Noble R. 2000. Viability, susceptibility to peroxidation and fatty acid composition of boar semen during liquid storage. Anim Reprod Sci 58: 99-111.

6. Chihuailaf RH, Contreras PA, Wittwer FG. 2002. Patogénesis del estrés oxidativo: consecuencias y evaluación en salud animal. Vet Méx 33: 265-283.

7. Córdova A et al. 2009. Estrés oxidativo y antioxidantes en la conservación espermática. Rev Complut Cs Vet 3: 1-38.

8. Domínguez AE et al. 2010. Improving the effect of incubation and oxidative stress on thawed spermatozoa from red deer by using different antioxidant treatments. Reprod Fertil Dev 22: 856-870.

9. El-Raey M, Badr MR, Rawash ZM, Darwish MG. 2014. Evidences for the role of melatonin as a protective additive during buffalo semen freezing. Amer J Anim \& Vet Sci 9: 252-262.

10. Hernández A et al. 2015. Actividad de las enzimas superóxido dismutasa (SOD) y glutatión peroxidasa (GSH-PX) del plasma seminal de cerdo en Veracruz, México. Act Iberoam Conserv Anim 6: 98-111.

11. Jang $\mathbf{H}$ et al. 2010. Ameliorative effects of melatonin against hydrogen peroxide-induced oxidative stress on boar sperm characteristics and subsequent in vitro embryo development. Reprod Domest Anim 45: 943-950. 
12. Johnson L, Weize K, Fiser P, Maxwell W. 2000. Storage of boar semen. Anim Reprod Sci 62:143-172.

13. Karimfar M et al. 2015. The protective effects of melatonin against cryopreservation-induced oxidative stress in human sperm. Inter J Imm Pathol \& Pharm 28: 69-76.

14. Lin D, Connor W, Wolf D, Neuringer M, Hachey D. 1993. Unique lipids of primate spermatozoa: desmosterol and docosahexaenoic acid. J Lipid Res 34: 491-499.

15. Marti E, Marti J, Muiño T, Cebrián J. 2008. Effect of the cryopreservation process on the activity and immunolocalization of antioxidant enzymes in ram spermatozoa. $J$ Androl 29: 459-467.

16. Martín D. 2013. Fisiología celular y calidad seminal durante la conservación del semen de porcino refrigerado. $D i$ sertación PhD, Fac.Cs.Vet.Univ.Extremadura, España, 234 p. https://dialnet.unirioja.es/servlet/tesis? codigo $=39557$

17. Mehrdad M, Mohammad J, Ahmadi Z, Saeed Z, Hamid K, Reza M. 2014. Effect of melatonin on in vitro stored semen quality and fertility of Fars indigenous roosters of Iran. Conf. Ass. Appl. Anim. Andrology and 12th Int. Symp. Spermatol. http://androfest.m.asnevents. com.au/schedule/session/4370/abstract/18408.

18. Membrillo A, Córdova A, Hicks J, Ivonne M, Martínez V, Valencia J. 2003. Peroxidación lipídica y antioxidantes en la preservación de semen. Rev INCI 28: 699-704.

19. Ohkawa H, Ohishi N, Yagi K. 1979. Assay for lipid peroxides in animal tissues by thiobarbituric acid reaction. Anal Biochem 95: 351-358.

20. Perumal P, Vupru K, Khate K. 2013. Effect of addition of melatonin on the liquid storage $5^{\circ} \mathrm{C}$ of mithun (Bos frontalis). Semen Inter J of Zool 13:1-10.

21. Perumal P, Chamuah J, Nahak A, Rajkhowa C. 2015. Effect of seasons on semen production, effect of melatonin on the liquid storage $\left(5^{\circ} \mathrm{C}\right)$ with correlated study of birth rate in mithun (Bos frontalis). As Pac J Reprod 4: 1-12.
22. Reiter R, Tan D, Manchester L, Qi W. 2001. Biochemical reactivity of melatonin with reactive oxygen and nitrogen species. Cell Biochem Biophys 34: 237-256.

23. Ressmeyer A et al. 2003. Antioxidant properties of the melatonin metabolite N1-acetyl-5-methoxykynuramine (AMK): scavenging of free radicals and prevention of protein destruction. Redox Rep 8: 205-213.

24. Sánchez R. Congelación de semen en porcino. 2007. http://www.3tres3.com/los-expertos-opinan/congelacionde-semen-en-porcino-historia-y-evolucion_1767/

25. Sampath H, Ntambi JM. 2005. Polyunsaturated fatty acid regulation of genes of lipid metabolism. Ann Rev Nutrit 25: 317-340.

26. Succu S, Berlinguer F, Pasciu V, Satta V, Leoni G, Naitana S. 2011. Melatonin protects ram spermatozoa from cryopreservation injuries in a dose-dependent manner. $J$ Pineal Res 50: 310-318.

27. Surai P, Brillard J, Speake B, Blesbois E, Seigneurin F, Sparks N. 2000. Phospholipid fatty acid composition, vitamin E content and susceptibility to lipid peroxidation of duck spermatozoa. Theriogenology 53: 1025-1039.

28. Tamayo M. 2013. La selección de sementales bovinos en Cuba. Calidad de la producción seminal en futuros sementales Holstein, relación con el desarrollo testicular. Redvet 14: 1 .

29. Tan D et al. 1998. A novel melatonin metabolite, cyclic 3-hydroxymelatonin: a biomarker of in vivo hydroxyl radical generation. Biochem Biophys Res Commun 253: 614620.

30. Wallin B, Rosengren B, Shertzer H, Camejo G. 1993. Lipoprotein oxidation and measurement of thiobarbituric acid reacting substances formation in a single microtiter plate: its use for evaluation of antioxidants. Anal Biochem 208: 10-15. 